\title{
Exaggerated Response of Systolic Blood Pressure to Cycle Ergometer
}

\author{
Young Joo Kim, PhD ${ }^{1}$, Heaja Chun, $\mathrm{PhD}^{2}$, Chul-Hyun Kim, $\mathrm{PhD}^{3}$
}

\begin{abstract}
${ }^{1}$ Department of Rehabilitation Medicine, Inje University Sanggye Paik Hospital, Seoul; ${ }^{2}$ Division of Physical Education, Soonchunhyang University, Cheonan; ${ }^{3}$ Sport Science Institute, Korea National Sport University, Seoul, Korea
\end{abstract}

\begin{abstract}
Objective The aim of this study is to exam the effects of exercise modes on the systolic blood pressure and ratepressure product during a gradually increasing exercise load from low to high intensity.

Methods Fifteen apparently healthy men aged 19 to 23 performed the graded exercise tests on cycle ergometer (CE) and treadmill (TM). During the low-to-maximal exercises, oxygen uptake $\left(\mathrm{VO}_{2}\right)$, heart rate (HR), systolic blood pressure (SBP) and rate-pressure product were measured.

Results CE had a significantly lower maximum $\mathrm{VO}_{2}$ than TM (CE vs. TM: $48.51 \pm 1.30$ vs. $55.4 \pm 1.19 \mathrm{~mL} / \mathrm{kg} / \mathrm{min}$; $\mathrm{p}<0.001)$. However, CE showed a higher maximum SBP $\left(\mathrm{SBP}_{\max }\right)$ at the all-out exercise load than TM (CE vs. TM: $170 \pm 2.4$ vs. $154 \pm 1.7 \mathrm{mmHg}$; $<0.001$ ). During the low-to-maximal intensity increment, the slope of the HR with $\mathrm{VO}_{2}$ was the same as $\mathrm{VO}_{2}$ increased in times of the graded exercise test of CE and TM (CE vs. TM: $2.542 \pm 0.100$ vs. $2.506 \pm 0.087 ; \mathrm{p}=0.26$ ). The slope of increase on $\mathrm{SBP}$ accompanied by $\mathrm{VO}_{2}$ increase was significantly higher in $\mathrm{CE}$ than in TM (CE vs. TM: $1.669 \pm 0.117$ vs. $1.179 \pm 0.063 ; \mathrm{p}<0.001)$.

Conclusion The SBP response is stronger in CE than in TM during the graded exercise test. Therefore, there is a possibility that CE could induce a greater burden on workloads to cardiovascular system in humans than TM.
\end{abstract}

Keywords Cardiovascular system, Cardiopulmonary exercise test, Ergometry, Systolic pressure, Hemodynamics

\section{INTRODUCTION}

The graded exercise test is a test that analyzes the change in the body's respiratory gas, electrocardiogram, or blood pressure (BP), which is induced by gradually

\footnotetext{
Received July 25, 2012; Accepted October 26, 2012

Corresponding author: Chul-Hyun Kim

Sport Science Institute, Korea National Sport University, 1239 Yangjaedaero, Songpa-gu, Seoul 138-763, Korea

Tel: +82-2-410-6692, Fax: +82-2-410-6945, E-mail: kimch37@gmail.com

(c) This is an open-access article distributed under the terms of the Creative Commons Attribution Non-Commercial License (http://creativecommons. org/licenses/by-nc/3.0) which permits unrestricted noncommercial use, distribution, and reproduction in any medium, provided the original work is properly cited.

Copyright @ 2013 by Korean Academy of Rehabilitation Medicine
}

increasing the workload with the use of a cycle ergometer (CE) or a treadmill (TM) [1]. This test can detect latent cardiovascular diseases and evaluate cardiovascular function [2]. It is particularly useful for evaluating the cardiopulmonary capacity of athletes and normal people, as well as for clinical practice, such as diagnosis and evaluation of cardiovascular disease, assessment of treatment effect, ascertainment of pathophysiology, and exercise prescription for rehabilitation [3-6]. To perform the graded exercise test, a protocol and mode of exercise testing should first be decided upon. The protocol for the exercise stress test consists of standardized gradual increments of exercise workload (oxygen uptake $\left[\mathrm{VO}_{2}\right]$ ) from the resting phase to the maximal workload $[1,2]$. 
An appropriate exercise protocol can be decided upon according to the absence or presence of disease, age, and physical activity level of the examinee $[7,8]$. The two major modes for the graded exercise test are CE and TM. TM involves a higher exercise load than CE. Since most people are more accustomed to walking than to riding a bicycle, TM is generally preferred over CE $[4,8,9]$.

Several protocols for the graded exercise test have been developed, and the observable physiological features of the two modes of exercise test (CE and TM) have been studied. The hemodynamic responses to TM and CE in the exercise tolerance test have established that $\mathrm{VO}_{2 \max }$ (maximum oxygen uptake) was higher in TM than in CE. Therefore, the level and mechanism of the hemodynamic response differ in modes of the graded exercise test [7-11].

The results of previous studies, however, were only about hemodynamic responses to modes of exercise testing under the maximal exercise workload. Moreover, the hemodynamic changes that result from gradual increments in the exercise load, from low to maximal intensity, in TM and CE have not been successfully compared. Particularly, the systolic blood pressure (SBP) response to increased heart rate (HR) and $\mathrm{VO}_{2}$ in TM and CE has not yet been clearly identified [7-13]. Reed [12] reported that SBPs at $40 \%$ and $60 \%$ HR reserve was higher in CE than in TM. Also, it is reported that SBP at the maximum workload was higher in CE than in TM [9]. These results indicate that the degree of increase in SBP on the CE is likely to be higher than the degree of increase in SBP on the TM. As the increased SBP in CE could increase the myocardial burden of patients with BP-related diseases such as ischemic cardiovascular disease, hypertension, and aneurysm, which may have an adverse effect [14], it should carefully be considered in the exercise test and exercise prescription. Thus, the information on the differences in the increment level of SBP between CE and $\mathrm{TM}$ is necessary for making an exercise prescription.

Previous studies on the differences of SBP between different modes of exercise testing were based, however, on the HRs instead of $\mathrm{VO}_{2}$ as the criterion for the exercise workload, and used uniform intensities of the exercise workload instead of gradually increasing the exercise load from low to maximal intensity. Therefore, not much information on BP has been seen in the hemodynamic responses (particularly SBP) to graded exercise increments in the exercise load $\left(\mathrm{VO}_{2}\right)$ from low to high inten- sity in CE and TM exercise.

This study hypothesized that the SBP and rate-pressure rate during cycle ergometer exercise could be higher than SBP and rate-pressure rate during a TM exercise at the given low-to-maximal exercise load expressed as $\mathrm{VO}_{2}$. The aim of this study is to exam the effects of exercise modes on the SBP and rate-pressure product (RPP) during a gradually increasing exercise load from low to high intensity.

\section{MATERIALS AND METHODS}

\section{Subjects}

Fifteen apparently healthy males who have no known diseases and live independently were recruited from a physical education college to take part in the study. All participants, who regularly performed graded exercise test at least two times a year for three years, were highly motivated and accustomed to the performance of exhaustive tests on both the bicycle ergometer and TM. The participation criteria were as follows: 1) nonsmoking; $2)$ age $\geq 19$ and $\leq 23 ; 3$ ) involvement in structured physical activity (i.e., regular moderate-to-high activity more than $240 \mathrm{~min} / \mathrm{wk}$ ) in the 8 months previous to the study; 4) absence of medication; and 5) absence of any medical or orthopedic condition aggravated by exercise. These inclusion criteria were selected for earning stable cardiovascular response to the graded exercise test. Participants gave written, informed consent after the experimental procedures and the associated risks as well as the benefits of participation were fully explained. All subjects were recruited through advertisements in local media. Sixty persons were interviewed on this study and 29 subjects voluntarily participated in this experiment. Among 29 subjects, 14 participants were dropped out because of unstable response to the graded exercise tests. Fifteen participants finally completed all the experiments of this study. Therefore, the compliance in the present study was $51.7 \%$. Written informed consent was obtained from all subjects. Institutional Review Board (IRB) approved the procedure and study design of this study (IRB serial no. 10-107).

\section{Exercise tests}

Participants were instructed to arrive at the laboratory in a rested state, at least 3-hour postprandial, to avoid 
strenuous exercise and alcohol in the 48 hours preceding a test session. They were weighted $(\mathrm{kg})$ and had their height $(\mathrm{cm})$ measured, and were fitted with a Heart Rate Monitor (Polar Electro, Kempele, Finland). Participants then completed a medical history and physical activity questionnaire. After allowing a ten-minute seated rest period, HR and BP were measured at rest by a Heart Rate Monitor and a mercury sphygmomanometer. Cardiopulmonary responses to exercise were measured during a graded CE model T300 (COSMED, Rome, Italy) and TM tests model Excalibur Sport (Lode B.V., Groningen, Netherlands) on two different days with an interval of one week in random sequence. Room temperature was $20^{\circ} \mathrm{C}-$ $24^{\circ} \mathrm{C}$ and relative humidity was $45 \%-55 \%$. TM testing was performed according to the Bruce protocol [1], which consists of three-minute increments of TM speed and incline, starting at a speed of $2.74 \mathrm{~km} / \mathrm{h}$ and a gradient of $10 \%$, increasing in gradient by $2 \%$ every 3 minutes in line with simultaneous increments of speed of 2.74, 4.02, $5.47,6.76,8.05$, and $8.85 \mathrm{~km} / \mathrm{h}$. The graded cycle ergometer testing used a Bruce ramp protocol that consists of continuous work rate increments [1]. With the pedaling cadence at $70 \mathrm{rpm}$, the work rate increased as a ramp of $35 \mathrm{~W} / \mathrm{min}$ to achieve maximal power output within 8 to 12 minutes of the test. Both test modes included a warmup period consisting of stretching exercises and a threeminute walk at $6.0 \mathrm{~km} / \mathrm{h}$ or a three-minute pedaling at 70 rpm with a load at $20 \mathrm{~W}$. The two protocols were comparable for gradually increasing loads and reaching the allout load even though the Bruce ramp protocol produced continuously increased loads because the sum of the three-minute increased loads at each three-minute-long stage were comparable with each stage of Brue protocol for TM [1,2]. During CE and TM tests, $\mathrm{VO}_{2}, \mathrm{HR}, \mathrm{BP}$, and the Borg-rated perceived exertion were recorded per minute starting from the baseline, starting from two and at maximal exercise. $\mathrm{VO}_{2}$, carbon dioxide production, and gas exchange ratio were measured on a breath-by-breath basis by a gas analyzer $\mathrm{K}_{4} \mathrm{~b}_{2}$ (COSMED). The HR was determined with a Heart Rate Monitor, and BP was measured with a mercury sphygmomanometer by a trained technician under supervision of a physician. The Borgrated perception of exertion was attained through direct communication by using the Borg scale. The RPP were obtained by multiplying the HR by SBP ( $R P P=H R \times S B P)$. The establishment of a maximal $\mathrm{VO}_{2}\left(\mathrm{VO}_{2 \max }\right)$ for each participant was evaluated against the criteria (a plateau in $\mathrm{VO}_{2}$, Borg-rated perceived exertion 19 or 20, HR within \pm 10 beats of age-related maximum and a respiratory exchange ratio $>1.15$ ) and confirmed if three of them were met. The gas analyzer was calibrated with mixed calibration gas every 5 exercise tests.

\section{Statistical analysis}

Based on the power analysis with G-power 3.0 program [14], it is estimated that at least 15 patients were requited to detect the mean differences in $\mathrm{VO}_{2}, \mathrm{HR}, \mathrm{SBP}$, and RPP between CE and TM, with a statistical power of $80 \%$, type I error of 0.05 , effects size of 0.7 and one-tailed test. To compensate for a possible exclusion, 29 subjects were first enrolled and after a dropout of 14 subjects (51.7\% of compliance). 15 subjects finally completed this study. Descriptive statistics were used for all the variables, and all values were presented as the means and standard errors. Comparisons of individual data between exercise modes were performed using Wilcoxon matched-pairs singed-ranks test. For estimating the increment ratios of the HR-to- $\mathrm{VO}_{2}$, SBP-to- $\mathrm{VO}_{2}$, RPP-to- $\mathrm{VO}_{2}$, and SBP-to-HR, the binary linear regression analysis was conducted with the model of ender. Independent variable was $\mathrm{VO}_{2}$ with the dependent variables of $\mathrm{HR}, \mathrm{VO}_{2}$, and RPP, respectively. Independent variable was also HR with SBP. With calculating the binary linear regression analysis, beta value was used for the slope of equation and constant value was used for $y$-intercept in the regression equation. F-value was identified for the significance of the equation model. The variance inflating factor (VIF) was calculated on the regressions of $\mathrm{VO}_{2}-\mathrm{HR}, \mathrm{VO}_{2}-\mathrm{SBP}, \mathrm{VO}_{2}-\mathrm{RPP}$, and $\mathrm{HR}-\mathrm{VO}_{2}$ and the values of VIF were 1.000 in all of the regression analyses. Pearson product moment coefficients (r) were used to assess the significance of relationships of hemodynamic response variables. The statistical significance level was set at $\mathrm{p}<0.05$ with the one-tailed test. All analyses were performed using SPSS ver. 11.0 (SPSS Inc., Chicago, IL, USA).

\section{RESULTS}

Fifteen healthy male participants all obtained the resting-to-maximal $\mathrm{VO}_{2}$ by reaching all-out intensity in a gradual stress test on CE and TM. Their general characteristics were shown at (Table 1), including age, physique, 
Table 1. Characteristics of subjects $(\mathrm{n}=15)$

\begin{tabular}{lcc}
\hline \multicolumn{1}{c}{ Characteristic } & Mean \pm SD & Range \\
\hline Age $(\mathrm{yr})$ & $21 \pm 1.3$ & $19-23$ \\
\hline Height $(\mathrm{cm})$ & $178.4 \pm 4.6$ & $168.3-186.0$ \\
Weight $(\mathrm{kg})$ & $76.4 \pm 7.3$ & $63.2-90.1$ \\
$\mathrm{BMI}\left(\mathrm{kg} / \mathrm{m}^{2}\right)$ & $24.0 \pm 1.7$ & $21.0-28.1$ \\
$\mathrm{HR}_{\text {rest }}(\mathrm{beat} / \mathrm{min})$ & $61 \pm 3.4$ & $53-66$ \\
$\mathrm{SBP}_{\text {rest }}(\mathrm{mmHg})$ & $105 \pm 5.8$ & $95-115$ \\
$\mathrm{DBP}_{\text {rest }}(\mathrm{mmHg})$ & $69 \pm 6.7$ & $52-80$ \\
$\mathrm{MAP}_{\text {rest }}(\mathrm{mmHg})$ & $81 \pm 5.4$ & $67-90$ \\
$\mathrm{RPP}_{\text {rest }}$ & $6,449 \pm 468$ & $5,782-7,260$ \\
\hline
\end{tabular}

Values are mean \pm standard deviation (SD).

$\mathrm{BMI}$, body mass index; $\mathrm{HR}_{\text {rest, }}$ resting heart rate; $\mathrm{SBP}_{\text {rest }}$, systolic blood pressure at rest; $\mathrm{DBP}_{\text {rest }}$, diastolic blood pressure at rest; $\mathrm{MAP}_{\text {rest }}$, mean arterial blood pressure at rest; $\mathrm{RPP}_{\text {rest }}$, rate-pressure products at rest.

(A)

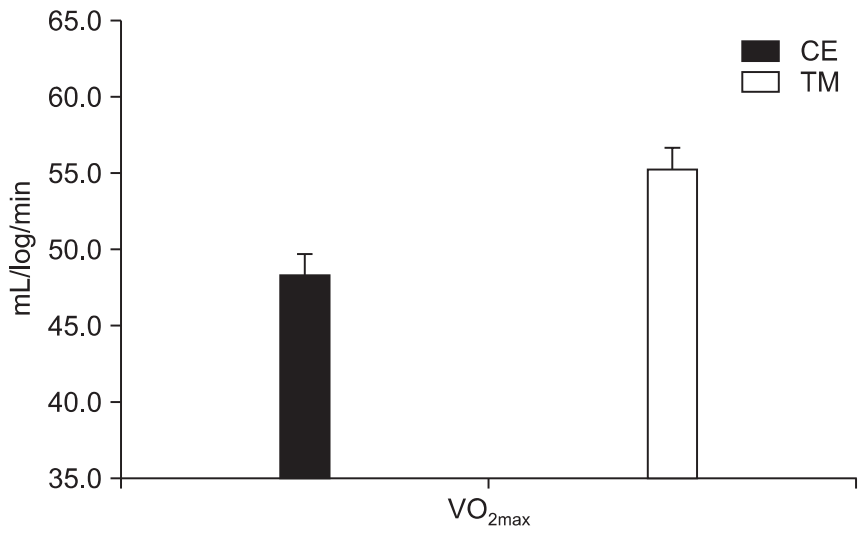

(C)

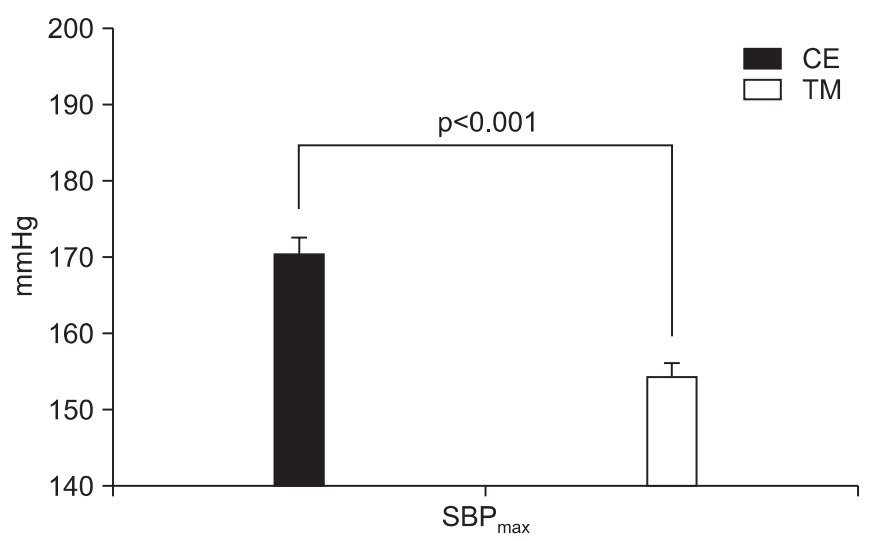

$\mathrm{HR}$, and $\mathrm{BP}$ at resting. As shown in (Fig. 1), the $\mathrm{VO}_{2 \max }$ on CE was significantly lower than that on TM (CE vs. TM: $48.51 \pm 1.30$ vs. $55.4 \pm 1.19 \mathrm{~mL} / \mathrm{kg} / \mathrm{min} ; \mathrm{p}<0.001)$. At this status, the maximum heart rate $\left(\mathrm{HR}_{\max }\right)$ was significantly lower in CE than TM (CE vs. TM: $177 \pm 2.4$ vs. $188 \pm 2.3$ beats/min; $\mathrm{p}<0.001)$, while maximum systolic blood pressure $\left(\mathrm{SBP}_{\max }\right)$ in $\mathrm{CE}$ was significantly higher than in TM (CE vs. TM: $170 \pm 2.4$ vs. $154 \pm 1.7 \mathrm{mmHg}, \mathrm{p}<0.001$ ), leading to a significantly higher load in the maximum rate-pressure product $\left(\mathrm{RPP}_{\max }\right)$ in CE compared to $\mathrm{RPP}_{\max }$ in TM (CE vs. TM: $30,149 \pm 678$ vs. $28,971 \pm 295$; $\mathrm{p}<0.05$ ). In other words, $\mathrm{CE}$ had lower $\mathrm{VO}_{2 \max }$ and $\mathrm{HR}_{\max }$ than $\mathrm{TM}$ at the level of aerobic maximal capacity, and showed high $\mathrm{SBP}_{\max }$ and $\mathrm{RPP}_{\max }$.

From the graded exercise tests on the CE and TM reaching from low intensity to maximal intensity, patterns of responses of HR, SBP, and RPP for gradually increasing

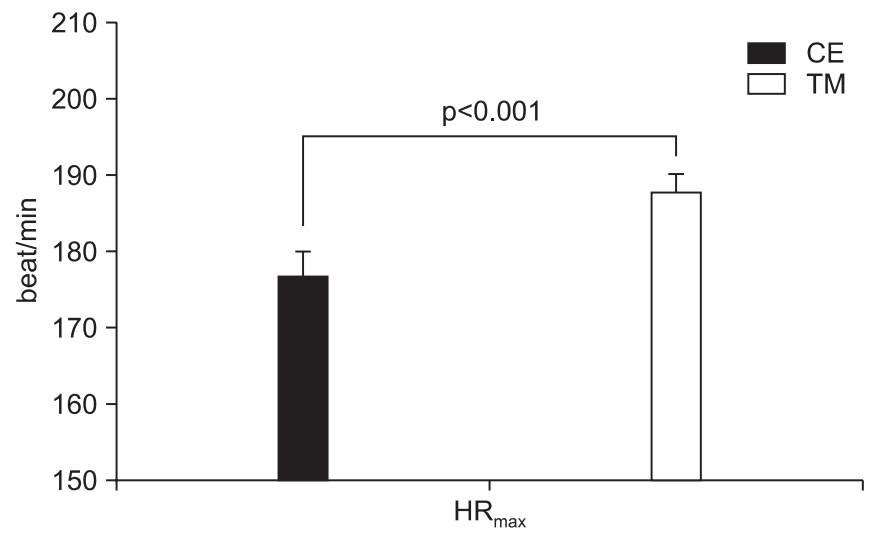

(D)

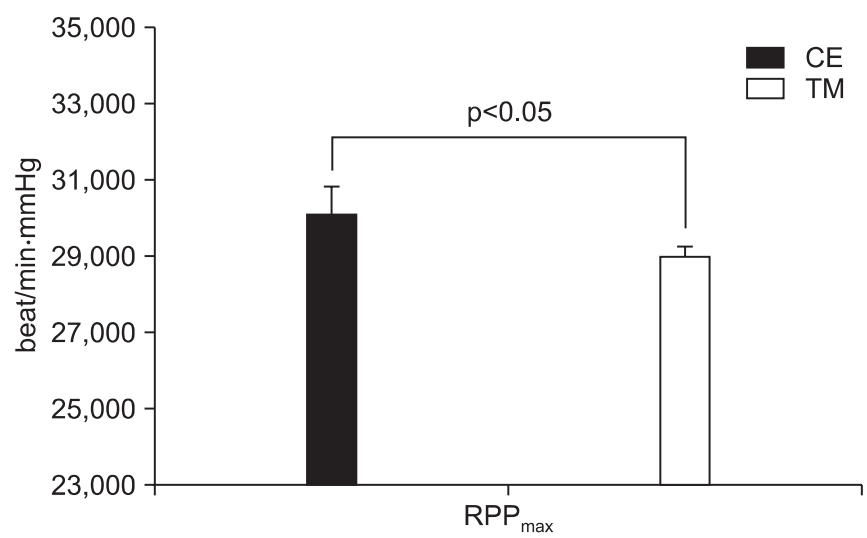

Fig. 1. Hemodynamic differences between cycle ergometer (CE) and treadmill (TM) at the maximum level of workload $(\mathrm{n}=15) . \mathrm{VO}_{2 \max }$, maximum oxygen uptake; $\mathrm{HR}_{\max }$, maximum heart rate; $\mathrm{SBP}_{\max }$, maximum systolic blood pressure; $\mathrm{RPP}_{\max }$, maximum rate-pressure product. 
(A)

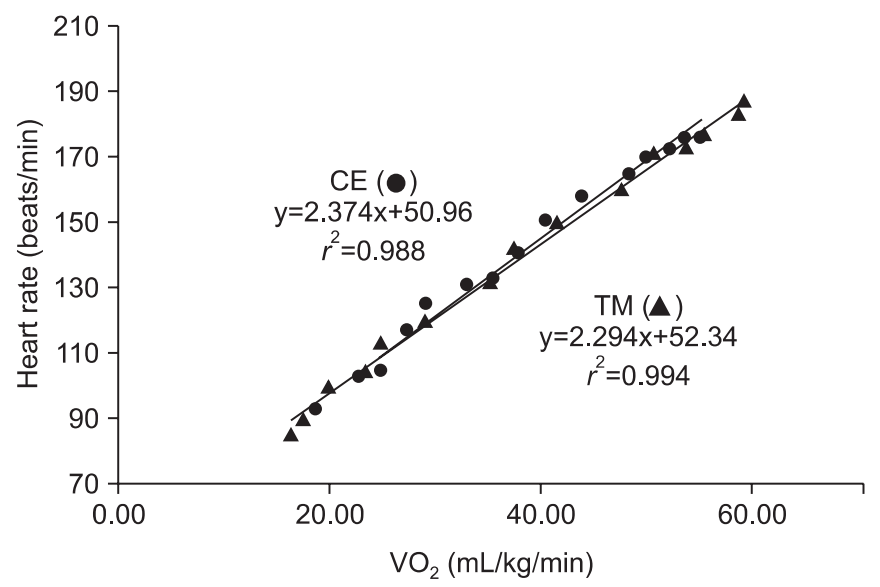

(C)

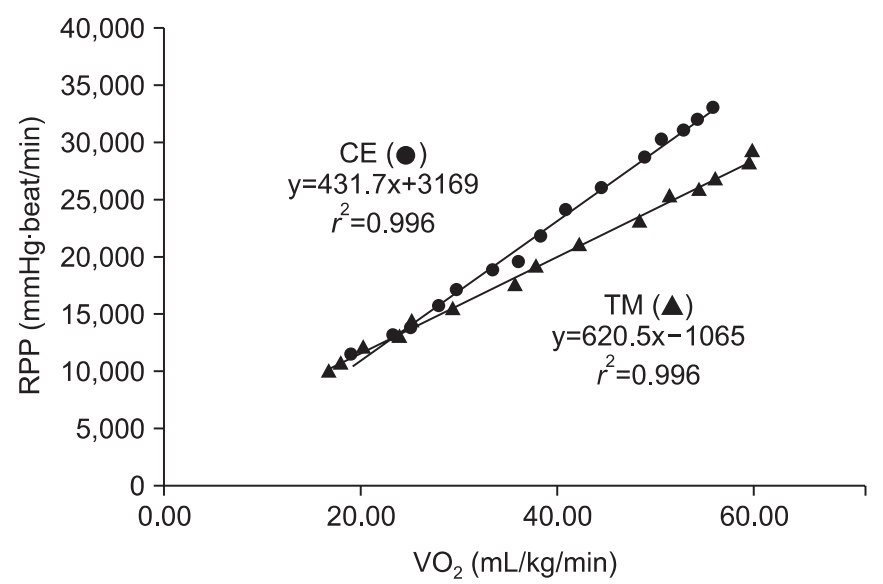

(B)

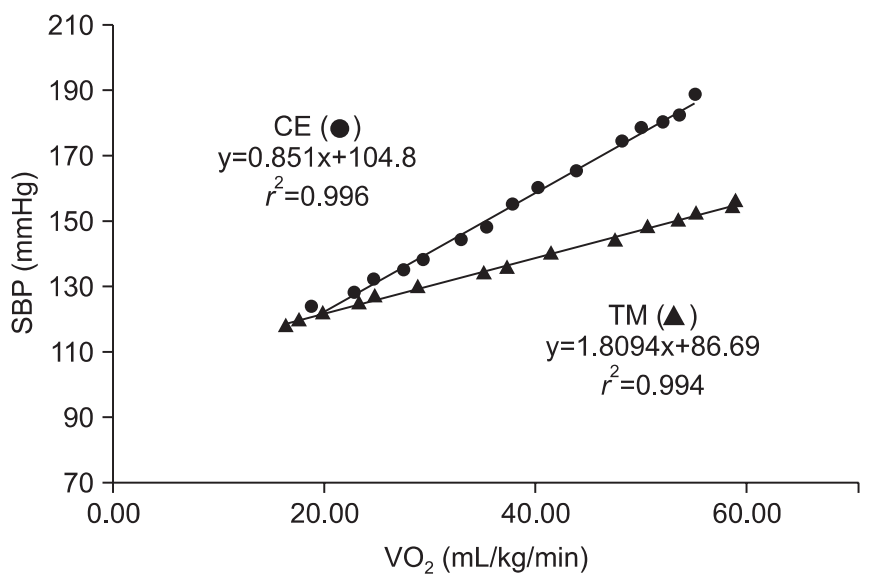

(D)

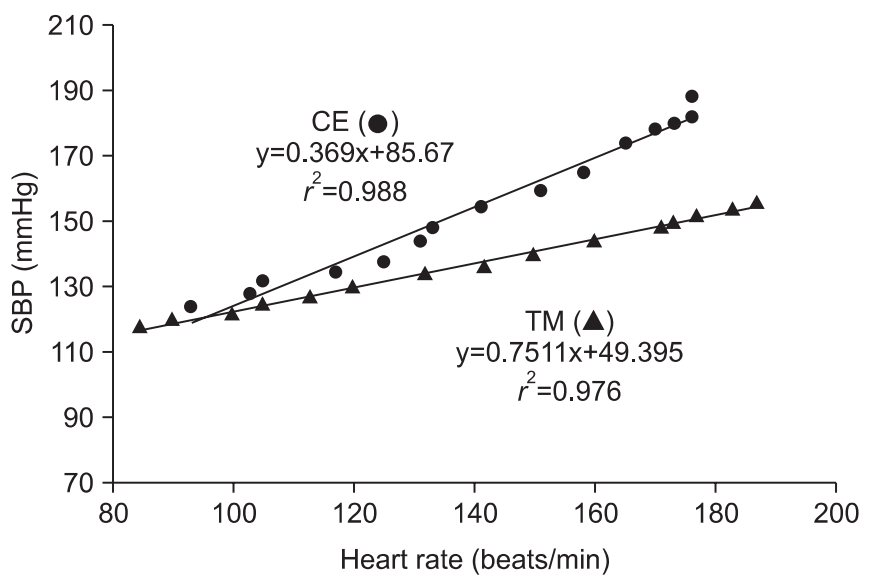

Fig. 2. An example of hemodynamic patterns of the heart rate, systolic blood pressure (SBP) and rate-pressure product (RPP) according to the work rates on cycle ergometer (CE) and treadmill (TM) during the gradual exercise test ( $n=1)$. $\mathrm{VO}_{2}$, oxygen uptake.

load $\mathrm{VO}_{2}$ are as in (Fig. 2). HR, SBP, and RPP proportionally increased as $\mathrm{VO}_{2}$ increased, but SBP and RPP of CE were higher than TM, except that HRs of CE and TM maintained the same level with each other in terms of the width and slope for the increase.

The results of a slope of the line from relation of $\mathrm{HR}$, SBP, and RPP with $\mathrm{VO}_{2}$ or SBP for fifteen participants (Fig. 3). While the increment of the $\mathrm{HR}-\mathrm{VO}_{2}$ appeared to be the same as $\mathrm{VO}_{2}$ increased in times of graded exercise test of CE and TM ( $p=0.26)$, the slope of SBP increase and RPP accompanied by $\mathrm{VO}_{2}$ increase (SBP- $\mathrm{VO}_{2}$ and $\mathrm{RPP}-\mathrm{VO}_{2}$, respectively) were all significantly higher in $\mathrm{CE}$ than in TM $(p<0.001)$. On the other hand, on the standard of the $\mathrm{HR}$, not $\mathrm{VO}_{2}$, for exercise load, SBP accompanied by the increase of exercise load (HR) elevated with a stiffer slope in CE than in TM $(p<0.001)$. In the end, SBP in times of gradually increasing exercise load for CE increased relatively a lot in TM, and RPP too elevated to a high level as a result.

\section{DISCUSSION}

In this study, two modes of exercise tests (CE and TM exercise) were performed with gradual increments in the exercise workload from low to maximal intensity, to identify the differences in the hemodynamic responses, particularly $\mathrm{VO}_{2}, \mathrm{HR}, \mathrm{SBP}$, and RPP during the low-tomaximal exercise. The results showed that $\mathrm{VO}_{2}, \mathrm{HR}, \mathrm{SBP}$, and RPP increased in positively direct proportion to the increase in the exercise workload in TM and CE. The 
(A)

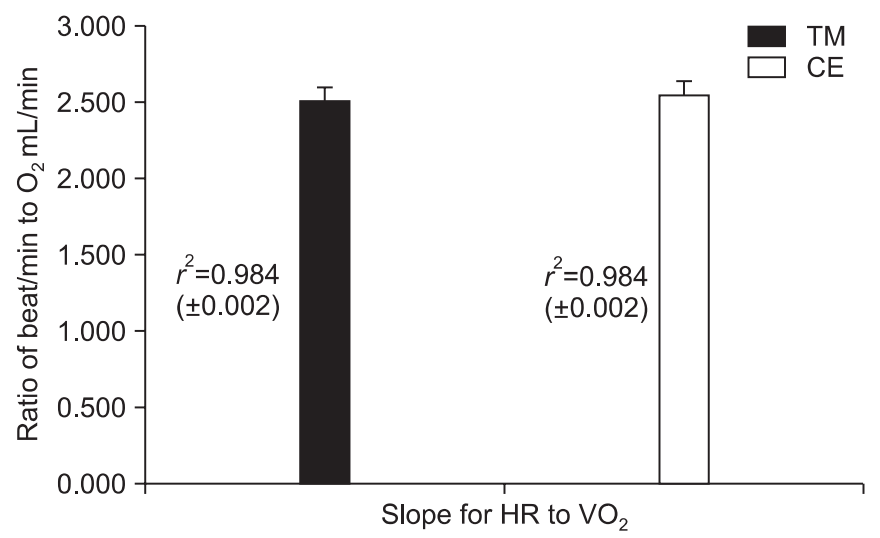

(C)

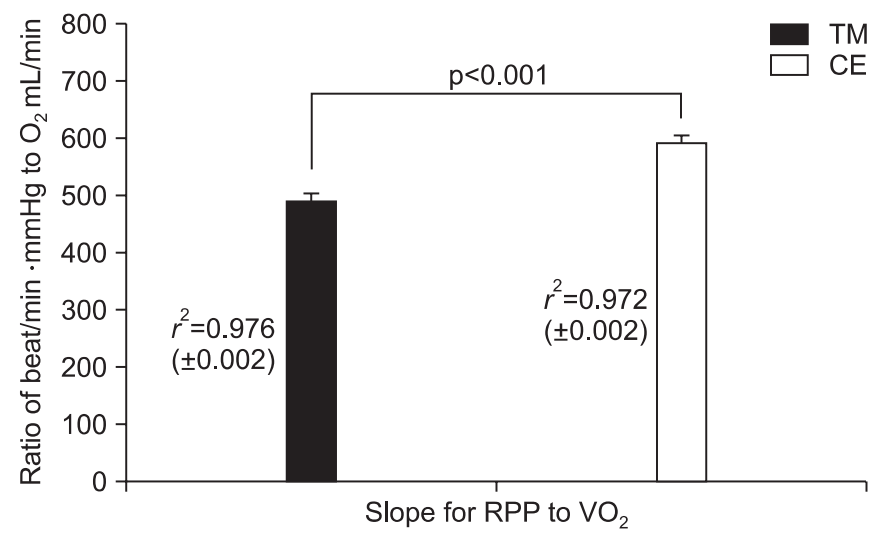

(B)

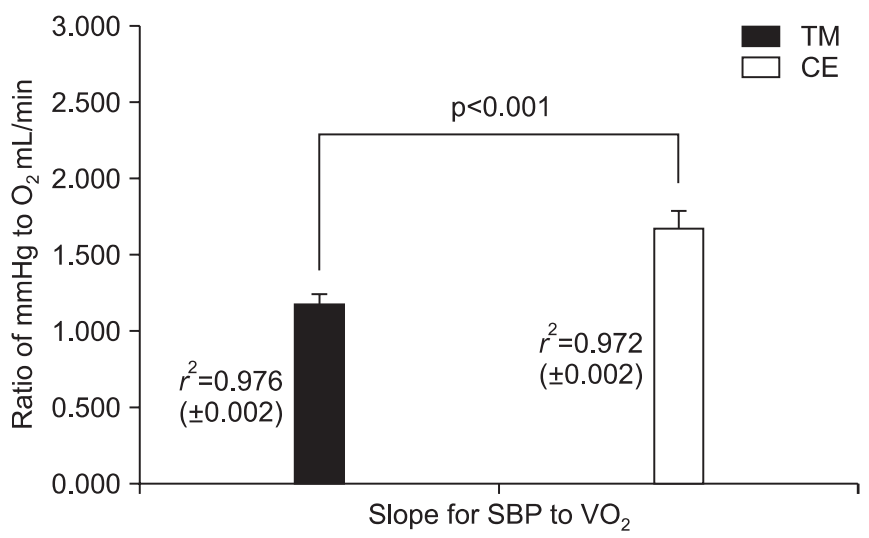

(D)

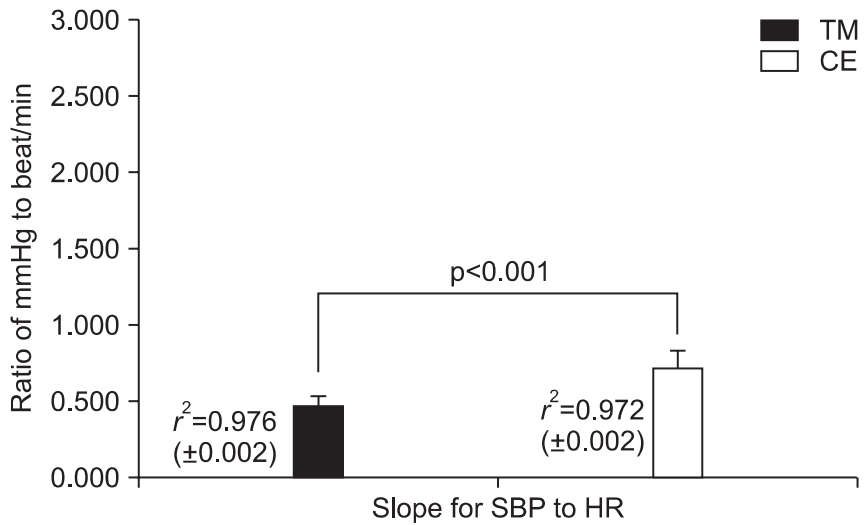

Fig. 3. The slope differences between cycle ergometer (CE) and treadmill (TM) exercise during the graded exercise test. (A) Slope for heart rate (HR) to oxygen uptake $\left(\mathrm{VO}_{2}\right)$ : $\mathrm{HR}$ is the $\mathrm{y}$-axis and $\mathrm{VO}_{2}$ is the $\mathrm{x}$-axis. (B) Slope for systolic blood pressure (SBP) to $\mathrm{VO}_{2}: \mathrm{SBP}$ is the $\mathrm{y}$-axis and $\mathrm{VO}_{2}$ is the $\mathrm{x}$-axis. (C) Slope of rate-pressure product (RPP) to $\mathrm{VO}_{2}$ : $\mathrm{RPP}$ is the $\mathrm{y}$-axis and $\mathrm{VO}_{2}$ is the $\mathrm{x}$-axis. (D) Slope for HR to SBP: HR is the $\mathrm{y}$-axis and SBP is the $\mathrm{x}$-axis. All values are mean \pm standard error.

slope of the increasing line in the hemodynamic variables with the increased $\mathrm{VO}_{2}$ differed, however, between CE and TM.

Conventionally, in the graded exercise tolerance tests, $\mathrm{VO}_{2 \max }$ differ most conspicuously in CE and TM. It has been reported that $\mathrm{VO}_{2 \max }$ was higher in $\mathrm{TM}$ than in $\mathrm{CE}$ $[15,16]$, and that the degree of difference varied from $6 \%$ to $25 \%$ [16]. These differences are considered attributable to the difference in examinees' ages and degrees of physical fitness, the type of exercise, and exercise test protocols. Consistent with the results of the previous studies, $\mathrm{VO}_{2 \max }$ in this study were significantly lower in $\mathrm{CE}$ than in TM. As the TM test can most accurately measure $\mathrm{VO}_{2 \max }$ and $\mathrm{HR}_{\max }$, the sensitivity in detecting myocardial ischemia is naturally higher in TM than in CE $[17,18]$.
In this study, $\mathrm{VO}_{2}$ was first used as the criterion for the increasing exercise workload, and the degrees of HR, SBP, and RPP responses to the CE and TM exercise were ascertained. HR increased to the same degree as the increase of $\mathrm{VO}_{2}$ in CE and TM; whereas SBP and RPP increased considerably more in CE than in TM for most of the lowto high-intensity exercise loads. The slopes of the line of $\mathrm{HR}-\mathrm{VO}_{2}$ with the gradual increase in the exercise workload in TM and CE were the same. The slopes of SBP and $\mathrm{RPP}$ with the increases in $\mathrm{HR}$ and $\mathrm{VO}_{2}$ were significantly higher in CE than in TM, though. As such, the increases in HR, SBP, and RPP had direct proportional relationships to the increase in $\mathrm{VO}_{2}$, and both SBP and RPP, but not HR, increased more in CE than in TM. Also, the SBP increase with the increase in $\mathrm{HR}$ was higher in CE than in 
TM. These results indicate that the BP response at a maximal exercise load is stronger in CE than in TM, which leads to higher RPP.

Balogun et al. [17] reported that the higher RPP in CE than in TM was attributable to the increased HR. In this study, however, the SBP increase was higher in CE than in TM when the same oxygen uptake and HR were used as criteria for the exercise load, and consequently, RPP also increased. This is a very interesting result that was not ascertained in the previous studies. A higher SBP in CE than in TM is observable in athletes. BP of endurance athletes during exercise can increase to $175 / 69 \mathrm{mmHg}$; whereas resistance athlete's BP can reach 480/350 mmHg at the maximum effort. Also, cycle athlete's BP increases to over $200 \mathrm{mmHg}$ during cycling exercise [19-22]. Cycling and rowing are both dynamic and static exercises. Fagard [22] reported that in a large-scale study of 947 elite athletes, cycling and rowing increased the inside diameter of the left ventricle and the thickness of the myocardial wall the most. The high SBP during cycling and rowing is the main cause of the increased inside diameter of the left ventricle and the increased thickness of the myocardial wall [22]. In a recent study of women with sedentary lifestyles, both their SBP and diastolic blood pressure were higher in the CE test than in TM when $40 \%$ and $60 \%$ of the target HR were used [12]. That study explained the reason for the higher $\mathrm{BP}$ in $\mathrm{CE}$ as the increase in the blood flow resistance in the muscle in CE under a similar workload, the reduced stimulation of the vagus nerve, and the increased tension in the muscle with increased exercise intensity [23-25].

Generally, an exercise prescription is made with a lower minimal load, and it is recommended that the exercise load is $85 \%$ of the target HR, according to the Karvonen et al. [26]. When the data obtained from the TM test are applied to the Karvonen et al. [26], normal people can exercise in CE without any problem. However, patients with ischemic cardiovascular disease, exercise-induced hypertension, and aneurysm may be subject to a higher myocardial burden in CE. Therefore, for them to be able to exercise in TM, the exercise intensity should be determined based on the exercise load test performed in TM; and likewise, for them to be able to exercise in CE, the exercise prescription should be made based on the HR obtained from the exercise load test performed in CE. The correlation between the change in $\mathrm{BP}$ with increased
HR in TM and CE should be identified in further studies; and based on such a correlation, an exercise prescription should be made using the formula for converting HR in TM to HR in CE, to obtain a similar myocardial burden.

Conventionally, the myocardial oxygen uptake $\left(\mathrm{MVO}_{2}\right)$ has been an effective marker of the coronary circulation response in relation to the myocardial metabolic demand. $\mathrm{MVO}_{2}$ can be measured from SBP and HR $(\mathrm{HR} \times \mathrm{SBP})$, and is generally defined as RPP or a double product $[27,28]$. It is reported that RPP is the most reliable marker of myocardial perfusion in patients with coronary arterial disease as well as in normal people. Many patients with chronic stable angina are likely to experience repetitive chest pain at the same RPP [5,29]. Moreover, the change in the ischemic ST segment occurs at certain RPPs, and the BP is likely to increase in patients with exercise-induced hypertension more significantly in CE than in TM. It was also reported that exercises that involve an increase in RPP without increasing HR to over 100 were the main reason for the rupture of aneurysm $[4,29]$. Beta-blockers, which are used to prevent such cardiovascular event and aneurysm, are known to be effective in reducing RPP $[3,5,29]$. Other research have also reported that cardiovascular disease patients, including hypertensive patients, showed a much more burden of RPP to the TM exercise at the intensity of $40 \%$ and $80 \%$ heart rate reserve (HRR) compared to the CE exercise at the same intensity of $40 \%$ and $80 \%$ HRR [30-32]. Thus, it was considered risky to apply data from TM to CE to make an exercise prescription for patients with ischemic cardiovascular disease, exercise-induced hypertension, or aneurysm. Although there has been no report that increased RPP due to increased SBP resulted in cardiovascular events more in $\mathrm{CE}$ than in TM at a maximal exercise load, clinical studies are required to ascertain this.

There was a limitation in this study. Even though the BP was measured by a single expertise in this study, the intra-rater reliability of BP measurements was not assessed. Therefore, some caution about measurement error on BP should be considered.

At the comparably given workloads ranging from the low to maximal intensity, the BP response is stronger in CE than in TM, which leads to higher RPP. Therefore, there is a possibility that CE could induce more burdens of workloads to cardiovascular system in humans. These findings could imply that people and patients with car- 
diovascular disease patients would do strenuous exercise at CE than in TM exercise.

\section{CONFLICT OF INTEREST}

No potential conflict of interest relevant to this article was reported.

\section{ACKNOWLEDGMENTS}

This study was supported by Korea National Sport University. We thank our colleagues for data collection and management at Korea National Sport University. We also thank all participants in this study.

\section{REFERENCES}

1. American College of Sports Medicine. ACSM's guidelines for exercise testing and prescription. 8th ed. Philadelphia: Lippincott Williams \& Wilkins; 2010.

2. Ellestad MH. Stress testing: principles and practice. 5th ed. New York: Oxford University Press; 2003.

3. Garber CE, Blissmer B, Deschenes MR, Franklin BA, Lamonte MJ, Lee IM, et al. American College of Sports Medicine position stand. Quantity and quality of exercise for developing and maintaining cardiorespiratory, musculoskeletal, and neuromotor fitness in apparently healthy adults: guidance for prescribing exercise. Med Sci Sports Exerc 2011;43:1334-59.

4. Fletcher GF, Balady GJ, Amsterdam EA, Chaitman B, Eckel R, Fleg J, et al. Exercise standards for testing and training: a statement for healthcare professionals from the American Heart Association. Circulation 2001;104:1694-740.

5. Mezzani A, Agostoni P, Cohen-Solal A, Corra U, Jegier A, Kouidi E, et al. Standards for the use of cardiopulmonary exercise testing for the functional evaluation of cardiac patients: a report from the Exercise Physiology Section of the European Association for Cardiovascular Prevention and Rehabilitation. Eur J Cardiovasc Prev Rehabil 2009;16:249-67.

6. Myers J, Prakash M, Froelicher V, Do D, Partington S, Atwood JE. Exercise capacity and mortality among men referred for exercise testing. $\mathrm{N}$ Engl J Med 2002;346:793-801.

7. Faulkner JA, Roberts DE, Elk RL, Conway J. Cardiovas- cular responses to submaximum and maximum effort cycling and running. J Appl Physiol 1971;30:457-61.

8. Hermansen L, Ekblom B, Saltin B. Cardiac output during submaximal and maximal treadmill and bicycle exercise. J Appl Physiol 1970;29:82-6.

9. Maeder M, Wolber T, Atefy R, Gadza M, Ammann $\mathrm{P}$, Myers J, et al. Impact of the exercise mode on exercise capacity: bicycle testing revisited. Chest 2005;128:2804-11.

10. Pollock ML, Foster C, Schmidt D, Hellman C, Linnerud AC, Ward A. Comparative analysis of physiologic responses to three different maximal graded exercise test protocols in healthy women. Am Heart J 1982;103:363-73.

11. Wicks JR, Sutton JR, Oldridge NB, Jones NL. Comparison of the electrocardiographic changes induced by maximam exercise testing with treadmill and cycle ergometer. Circulation 1978;57:1066-70.

12. Reed J. Blood pressure responses of sedentary African American women during cycle and treadmill exercise. Ethn Dis 2007; 17:59-64.

13. Foster C, Gaeckle T, Braastad R, Schmidt DH, Port SC. First-pass radionuclide angiography during bicycle and treadmill exercise. J Nucl Cardiol 1995;2:485-90.

14. Mundal R, Kjeldsen SE, Sandvik L, Erikssen G, Thaulow E, Erikssen J. Exercise blood pressure predicts cardiovascular mortality in middle-aged men. Hypertension 1994;24:56-62.

15. Glassford RG, Baycroft GH, Sedgwick AW, Macnab RB. Comparison of maximal oxygen uptake values determined by predicted and actual methods. J Appl Physiol 1965;20:509-13.

16. Myers J, Buchanan N, Walsh D, Kraemer M, McAuley P, Hamilton-Wessler M, et al. Comparison of the ramp versus standard exercise protocols. J Am Coll Cardiol 1991;17:1334-42.

17. Balogun MO, Sulyman BO, Akinwusi PO. A comparison of the cardiovascular responses to treadmill and bicycle ergometer exercise in healthy male Nigerians. Afr J Med Med Sci 1997;26:27-30.

18. Yamakado T, Kasai A, Masuda T, Futagami Y, Kawasaki A, Zhang Y, et al. Exercise-induced coronary spasm: comparison of treadmill and bicycle exercise in patients with vasospastic angina. Coron Artery Dis 1996;7:819-22.

19. MacDougall JD, Tuxen D, Sale DG, Moroz JR, Sutton 
JR. Arterial blood pressure response to heavy resistance exercise. J Appl Physiol 1985;58:785-90.

20. Mitchell JH, Haskell WL, Raven PB. Classification of sports. Med Sci Sports Exerc 1994;26(10 Suppl):S242-5.

21. Palatini P, Mos L, Mormino P, Munari L, Del Torre M, Valle F, et al. Intra-arterial blood pressure monitoring in the evaluation of the hypertensive athlete. Eur Heart J 1990;11:348-54.

22. Fagard R. Athlete's heart. Circulation 2001;103:E28-9.

23. Blomqvist CG, Lewis SF, Taylor WF, Graham RM. Similarity of the hemodynamic responses to static and dynamic exercise of small muscle groups. Circ Res 1981;48(6 Pt 2):187-92.

24. Clarys JP, Cabri J, Gregor RJ. The muscle activity paradox during circular rhythmic leg movements. J Sports Sci 1988;6:229-37.

25. Tulppo MP, Makikallio TH, Laukkanen RT, Huikuri HV. Differences in autonomic modulation of heart rate during arm and leg exercise. Clin Physiol 1999;19:2949.

26. Karvonen MJ, Kentala E, Mustala O. The effects of training on heart rate; a longitudinal study. Ann Med Exp Biol Fenn 1957;35:307-15.

27. Nelson RR, Gobel FL, Jorgensen CR, Wang K, Wang Y, Taylor HL. Hemodynamic predictors of myocardial oxygen consumption during static and dynamic exercise. Circulation 1974;50:1179-89.

28. Gobel FL, Norstrom LA, Nelson RR, Jorgensen CR, Wang Y. The rate-pressure product as an index of myocardial oxygen consumption during exercise in patients with angina pectoris. Circulation 1978;57:54956.

29. Gajulapalli RD, Aneja A, Rovner A. Cardiac stress testing for the diagnosis and management of coronary artery disease: a reference for the primary care physician. South Med J 2012;105:93-9.

30. Kim YJ, Kim C, Ahn JK, Lim SW, Kim EK, Shin YO, et al. Comparison of myocardial oxygen uptake and blood pressure in myocardial infarction patients response to treadmill and bike exercise therapy. Korean J Sports Med 2002;20:15-21.

31. Kim C, Kim YJ. Comparison of myocardial oxygen demand and rate of perceived exertion according to the modes of exercise in ischemic heart disease. J Korean Acad Rehabil Med 2009;33:572-7.

32. Kim YJ, Kim CH. Cardiovascular and perceived exertion response to treadmill running and cycle ergometer exercise in reponsder and nonreponder acute coronary syndrome patients. J Life SCI 2008;18:126370. 